ORIGINAL ARTICLE

\title{
The Effect of Hypothyroidism on the Development and course of Heart Failure
}

\author{
ROMAN EVGENYEVICH TOKMACHEV ${ }^{1}$, ANDREY VALERIEVICH BUDNEVSKY ${ }^{2}$, ANDREY YAKOVLEVICH \\ KRAVCHENKO ${ }^{3}$, YANINA SERGEEVNA SHKATOVA ${ }^{4}$, TATIANA ALEXANDROVNA CHERNIK ${ }^{5}$, YULIA ANDREEVNA \\ KRASNIKOVA ${ }^{3}$ \\ ${ }^{1} \mathrm{MD}, \mathrm{PhD}$, doctoral candidate of the Department of Internal Medicine, Voronezh Burdenko State Medical University, Voronezh, Russian \\ Federation. \\ ${ }^{2} \mathrm{MD}$, PhD, Professor, Vice-Rector for Research and Innovation, Honored Inventor of the Russian Federation, Professor of the Department \\ of Internal Medicine, Voronezh Burdenko State Medical University, Voronezh, Russian Federation. \\ ${ }^{3} \mathrm{MD}, \mathrm{PhD}$, Professor of the Department of Internal Medicine, Voronezh Burdenko State Medical University, Voronezh, Russian Federation. \\ ${ }^{4} \mathrm{MD}$, Postgraduate student of the Department of Internal Medicine, Voronezh Burdenko State Medical University, Voronezh, Russian \\ Federation. \\ ${ }^{5} \mathrm{MD}$, Postgraduate student of the Department of Internal Medicine, Voronezh Burdenko State Medical University, Voronezh, Russian \\ Federation. \\ ${ }^{6} \mathrm{MD}$, Postgraduate student of the Department of Internal Medicine, Voronezh Burdenko State Medical University, Voronezh, Russian \\ Federation. \\ Correspondence to Roman Evgenyevich Tokmachev Address: Moskovskiy pr-t street 141, 29, Voronezh, Russia, P hone: +79003003013. \\ mail: r-tokmachev@mail.ru
}

\begin{abstract}
Thyroid gland dysfunction is a risk factor for development of cardiovascular diseases. From all types of thyroid gland dysfunction hypothyroidism is the most common. Increasing of thyroid-stimulating hormone level can influence the clinical course of chronic heart failure (CHF) because of its extrathyroid effects - namely - influence on lipide metabolism, endothelial function of vessels and blood pressure. Also CHF itself can make negative impact on a thyroid gland functioning, therefore pathophysiological "vicious circle" is formed. Manifest hypothyroidism is associated with an increased risk of general and cardiovascular mortality and frequency of hospitalizations of CHF patients. Association between subclinical hypothyroidism and heart failure is less studied. Figuring out the mechanisms of influence of thyroid gland hypofunction on systolic and diastolic function of myocardium may be important for effective treatment of heart failure and improvement of clinical outcomes.

MeSH words: heart failure; hypothyroidism; thyroid-stimulating hormone (TSH); review.
\end{abstract}

\section{INTRODUCTION}

The prevalence of heart failure (HF) in developed countries is $1-2 \%$ among the adult population, increasing to $10 \%$ or more among people over 70 years old ${ }^{1}$. Among people over 65 years old with newly diagnosed dyspnea during exercise, every sixth person is diagnosed with heart failure, mainly with preserved left ventricular ejection fraction (LV $\mathrm{EF})$. The risk of $\mathrm{HF}$ at age 55 is $55 \%$ for men and $28 \%$ for women ${ }^{1}$. Recent data from pilot studies of the European Society of Cardiology on HF show that the mortality rate over a 12-month period from all causes for hospitalized patients with $\mathrm{HF}$ is $17 \%$, and for outpatients with $\mathrm{HF} 7 \%$, and hospitalization rates are $44 \%$ and $32 \%$ respectively. Most of the deaths in patients with HF (both hospitalized and outpatient) are associated with cardiovascular causes, mainly due to sudden cardiac death and worsening of $\mathrm{HF}^{2}$. According to the epidemiological study EPOCH-CHF, conducted in the Russian Federation, the prevalence of chronic heart failure (CHF) I-IVfunctional class in 2002 was $7 \%$ (7.9 million people). At the same time, the prevalence of CHF in the population is growing by an average of 1.2 people per 1000 population per year. The one-year mortality rate of patients with clinically expressed CHF reaches $12 \%$, i.e. in one year in the Russian Federation, up to 612 thousand patients with CHF die [3]. A number of studies have noted that the survival rate of patients with newly diagnosed CHF is $63 \%$ in the first year and $35 \%$ within 5 years [4]. Thus, CHF is one of the main causes of hospitalization and mortality.
The pathogenesis of HF is very complex and poorly understood. The activation of neuroendocrine mechanisms plays an important role in the progression of $\mathrm{HF}^{5,6}$. Hypothyroidism, defined as an increase in thyroidstimulating hormone (TSH) levels with a reduced level of triiodothyronine (T3) and thyroxine (T4), is widespread in the general population ${ }^{7}$. A review by Wickham showed that the incidence of overt hypothyroidism is 4.1 per 1000 per year for women and 0.6 per 1000 per year for men, and the overall prevalence is 14-19 per 1000 for women and less than 1 per 1000 for men. The same study showed that the prevalence of subclinical hypothyroidism (SCH) is 75 per 1000 for women and 28 per 1000 for men. They can develop a manifest form of hypothyroidism with a frequency of about $2-5 \%$ per year ${ }^{8}$.

Dysfunction of the thyroid gland (TG) is a risk factor for the development of a number of cardiovascular diseases $(C V D)^{9-13}$. A growing number of studies indicate that thyroid hormones (TH) have a significant effect on the cardiovascular system (CVS): myocardial contractility, heart rate, diastolic heart function, systemic vascular resistance (SVR), blood pressure (BP) ${ }^{19,10,23}$. Moreover, they interact with the sympathetic nervous system, the reninangiotensin-aldosterone system, indirectly affecting the heart and vascular function. Accordingly, hypothyroidism can have an adverse effect on the course of $C V D^{13}$. $S C H$ can also act as one of the risk factors for CVD, increasing the SVR, increasing the stiffness of the vascular wall and contributing to the development of endothelial 
dysfunction ${ }^{10,11}$. SCH can worsen age-related changes in the myocardium, which leads to an increased risk of progression of HF, and may also represent an independent risk factor for the development and progression of HF, including $\mathrm{CHF}^{14}$. Long-term manifest or $\mathrm{SCH}$ can affect lipid metabolism, contributing to an increase in blood low density lipoproteins (LDL) and B-apolipoproteins. Moreover, it can have a negative effect on other risk factors for atherothrombotic diseases: the level of C-reactive protein and homocysteine in the blood plasma, vascular endothelial function, blood pressure and coagulation parameters. Manifest hypothyroidism is usually detected promptly and corrected by the administration of levothyroxine, however, studies of its relationship to cardiovascular risk are mostly single-stage and using a small number of patients. SCH is not only more common than manifest, but also remains untreated for a long period of time. This, in turn, may increase cardiovascular risk, as shown in some studies ${ }^{17}$.

The American College of Cardiology and the American Heart Association recommend testing thyroid function in patients with HF, especially the TSH level, in order to identify hypothyroidism as a major or predisposing factor in the development of $\mathrm{HF}^{18}$. TSH levels are likely to influence the course of $\mathrm{HF}$, as it has been found to have "extrathyroid" effects. TSH can stimulate the production of interleukin-6 (IL-6) and tumor necrosis factor (TNF) in vitro, and may be involved in the regulation of vascular function by increasing nitrogenous acid metabolites in the body $A$ possible link between an increase in TSH levels and inflammation, as well as oxidative stress, has been suggested $^{19}$.

Interest in the role of triglycerides in the development of $\mathrm{HF}$ has been increasing recently, mainly due to two reasons: growing evidence of the physiological and homeostatic effects of triglycerides on the CVS; the emergence of evidence that altered $\mathrm{TH}$ metabolism affects CVS as a strong predictive marker of heart disease, especially in the presence of HF [20]. Experimental studies show that hypothyroidism contributes to impaired LV diastolic function by slowing down myocardial relaxation and decreasing ventricular filling. There is also a dysfunction of LV systolic function, both at rest and during exercise $^{21}$. In addition to the adverse effects of thyroid hormone deficiency on the onset and progression of CHF, HF itself can have a negative effect on thyroid function, resulting in the formation of a pathophysiological "vicious circle" involving thyroid hypofunction, leading to many clinical manifestations of HF, including electrical instability of the myocardium. Accordingly, the interruption of this pathophysiological chain may be important for the effective treatment of $\mathrm{HF}^{22-23}$

In 2012 B. Gencer et al. revealed an increased risk of HF progression in patients with hypothyroidism ${ }^{12}$. However, only a small number of studies have been devoted to studying the effect of hypothyroidism on overall mortality in patients with CVD and, in particular, with CHF. Some studies have described an increased risk of all-cause mortality in patients with a combination of CHF and hypothyroidism ${ }^{19,26}$, while others have not ${ }^{27}$. In addition, in most studies, the number of patients with hypothyroidism was small. Thus, the relationship between hypothyroidism and clinical outcomes in patients with CHF remains poorly understood. In 2015, Ning et al. conducted a meta-analysis [20], the aim of which was to identify the relationship between hypothyroidism and mortality (general and cardiovascular) and / or hospital admissions due to worsening CVD. The meta-analysis included 13 studies that included 20446 patients (of which 2306 patients with hypothyroidism). The incidence of hypothyroidism averaged $11.4 \%$ (ranged from 4-24\%). The analysis demonstrated that hypothyroidism is associated with a significant increase in the risk of all-cause mortality, cardiovascular mortality, and hospital admissions for patients with HF. In addition, this association persisted after excluding patients who initially used $\mathrm{TH}$ or amiodarone in therapy.

There is conflicting evidence on the effect of $\mathrm{SCH}$ on mortality. In 2008, Razvi et $\mathrm{al}^{28}$ and in 2007 Ochs et $\mathrm{al}^{29}$ found that the overall mortality rate is higher in the group of patients with $\mathrm{SCH}$ compared with euthyroid patients. However, a 2010 meta-analysis by Rodondi et al. Indicates a slight increase in the risk of all-cause mortality in patients with $\mathrm{SCH}^{30}$. lervasi et al. (2007) found an increase in mortality, especially from ischemic heart disease, in patients with SCH and CVD compared with euthyroid patients. These findings can be explained by the fact that $\mathrm{TH}$ play an important role in the regulation of myocyte morphology, metabolism, function and cellular response to stress, increasing the heart's tolerance to ischemia.

In a 2018 Kevin Ro et al studied the relationship of thyroid status, as measured by plasma TSH, with the risk of hospitalization among patients receiving care at a large university health care center in 1990-2015. Thyroid status was classified as either hypothyroidism or euthyroidism (TSH> 4.7 versus $0.3-4.7 \mathrm{mIU} / \mathrm{L}$, respectively). The relationship between thyroid status and hospitalization risk stratified by cardiovascular status was studied using multivariate Cox models. Among the 52,856 patients who met the eligibility criteria, 49,791 (94.2\%) had euthyroidism and 3065 (5.8\%) had hypothyroidism. In analyzes stratified by congestive HF status compared with euthyroidism, hypothyroidism was associated with a higher risk of hospitalization in individuals with CHF, but a slightly lower risk in individuals without CHF (adjusted hazard ratio [aHR] $=1,86$ [confidence interval $(\mathrm{Cl}) 1.17-2.94$ ] and $\mathrm{RR}=0.95$ [Cl 0.92-0.99], respectively; $p=0.006$ ). Thus, the authors concluded that hypothyroidism is associated with a higher risk of hospitalization among patients with concomitant cardiovascular diseases and further research is needed to determine whether correction of thyroid status with hormone replacement therapy improves the risk of hospitalization in this population ${ }^{31}$.

Also Penn Heart Failure Study by Lakshmi Kannan et al (2018) is requiring attention. In a large cohort of outpatients (1365 participants) with preexisting heart failure, the prevalence of thyroid dysfunction and its association with cardiovascular outcomes, the association between thyroid dysfunction and NYHA functional HF class, atrial fibrillation, and composite endpoint of placement of a left ventricular assist device, heart transplantation or death were examined. heart transplant or death. The median age of the subjects was $57.35 \%$ were women, and most of them had NYHA class II (45\%) or III 
$(32 \%)$. The study reflects that more severe $\mathrm{HF}$ is associated with higher TSH concentrations, higher free thyroxine (FT4) and lower total triiodothyronine (TT3) concentrations ( $P<0.001$ in all models). Atrial fibrillation was positively associated only with higher levels of FT4 ( $\mathrm{P} \leq 0.01$ for all models). In adjusted models, subclinical hypothyroidism compared with euthyroidism (TSH 4.5119.99 mIU / L with normal FT4) was associated with an increased risk of the combined endpoint (HR 1.82; 95\% C, 1.27-2.61; $\mathrm{R}=0.001$ ) and in the subgroup with $\mathrm{TSH} \geq 7.00$ mIU / L (HR 3.25; 95\% Di, 1.96-5.39; P <0.001). An isolated low T3 was also associated with a composite endpoint (HR 2.12; 95\% Cl 1.65-2.72; $\mathrm{P}<0.001)$. In patients with preexisting HF, subclinical hypothyroidism with $\mathrm{TSH} \geq 7 \mathrm{mIU} / \mathrm{L}$ and an isolated low T3 level is associated with a poor prognosis. Clinical trials are needed to investigate the therapeutic effects of T4 and T3 administration in patients with heart failure [32].

The analysis of the literature suggests that hypothyroidism in manifest or subclinical forms is quite common among the comorbidities that can affect the prognosis of HF [33-35]. The differences in these groups of patients in terms of overall mortality can be explained by the heterogeneity of the groups by sex, age, degree of TSH level change and duration of follow-up.

Thus, further research is needed to assess the effect of $\mathrm{SCH}$ on the clinical course of HF using well-developed patient sampling criteria.

Disclaimer: views expressed in the submitted article belong to the authors and not to the university.

Authors report no conflict of interests.

\section{REFERENCES}

1. Ponikowski P., Adriaan A. Voors, Stefan D. Anker, HéctorBueno, John G. F. Cleland, Andrew J. S. Coats. 2016 ESC Guidelines for the diagnosis and treatment of acute and chronic heart failure. Eur Heart J. 2016; 37 (27): 2129-2200.

2. Maggioni AP., Dahlström U., Filippatos G., Chioncel O., Leiro MC., Drozdz J.EUR Observational Research Programme: regional differences and 1-year follow-up results of the Heart Failure Pilot Survey (ESC-HF Pilot). Eur J Heart Fail. 2013; 15: 808-817.

3. MareevV.Yu., Ageev F.T., Arutyunov G.P., Koroteyev A.V., MareevYu.V., Ovchinnikov A.G., et al. National references of $\mathrm{OCCH}, \mathrm{PKO}$ and PHMOT on diagnostics and treatment of HCF (the fourth revision). Are approved on the Congress of OSSN on December 7, 2012, on Board of OSSN on March 31,2013 and the Congress of RKO on September 25, 2013. Heart failure.2013; 14 (7): 81. (in Russian).

4. Liu L., Eisen HJ. Epidemiology of heart failure and scope of the problem. Cardiol Clin. 2014; 32: 1-8.

5. Tokmachev, R.E., Budnevsky, A.V., Kravchenko, A.Y. The role of inflammation in the pathogenesis of chronic heart failure. Terapevticheskii Arkhiv, 2016; 88(9): 106-110.

6. Tokmachev R. E.; Budnevsky A., V; Kravchenko A. Ya. The Possibility of Non-Pharmacological Methods in Increasing Clinical Efficiency of Treating Patients with Chronic Heart Failure and Metabolic Syndrome. Research journal of pharmaceutical biological and chemical sciences. 2017; 8(6): 832-839.

7. Flynn R., MacDonald T., Morris A. et al. The thyroid epidemiology, audit, and research study: thyroid dysfunction in the general population. J Clin Endocrinol Metab. 2004; 89: 3879-3884.
8. Huber G., Staub J.J., Meier C., Mitrache C., Guglielmetti M., Huber P. et al. Prospective study of the spontaneous course of subclinical hypothyroidism: prognostic value of thyrotropin, thyroid reserve, and thyroid antibodies. J. Clin. Endocrinol. Meatab.2002; 87: 3221-3226.

9. Feskova A.A., Kravchenko A.Ja. Features of a clinical course of a hypertension against the background of a subclinical hypofunction of a thyroid gland.The Systemic analysis and management in biomedical systems. 2015; 14 (3): 473-476. (in Russian).

10. Gencer B., Collet TH, Virgini V. et al. Subclinical thyroid dysfunction and the risk of heart failure events: an individual participant data analysis from 6 prospective cohorts. Circulation. 2012; 126: 1040-1049.

11. Agata Bielecka-Dabrowa, Breno Godoy, Tsuyoshi Suzuki, Maciej Banach,Stephan von Haehling. Subclinical hypothyroidism and the development of heart failure:an overview of risk and effects on cardiac function. Clinical Research in Cardiology. 2019; 108:225-233.

12. Verbovoj A.F., Sharonova L.A., Kosareva O.V., Verbovaja N.I., Dolgih Ju.A. Hypothyroidism and cardiovascular system. Clinical Medicine. 2016; 94(7): 497-503. (in Russian).

13. Triggiani V., lacoviello M. Thyroid disorders in chronic heart failure: from prognostic set-up to therapeutic management. Endocr Metab Immune Disord Drug Targets. 2013; 13: 22 37.

14. Kravchenko A.Ja., Budnevskij A.V., Feskova A.A., Kalinichenko A.E. Some pathogenetic aspects of development of cardiovascular diseases at the minimum deficiency the tireoidnykh of hormones. The System analysis and management in biomedical systems. 2016; 15(1):14-17. (in Russian).

15. Vincenzo Triggiani and Massimo lacoviello et al. Thyroid Disorders in Chronic Heart Failure: From Prognostic Set-up to Therapeutic Management. Metabolic \& Immune Disorders Drug Targets. 2013; 13: 22-37.

16. Giuseppe Pasqualetti. «Is Subclinical Hypothyroidism a Cardiovascular Risk Factor in the El-derly?» Department of Clinical and Experimental Medicine, University of Pisa, Via Roma 67, 56126 Pisa, Italy. 2012.

17. Squizzato A., Romualdi E., Buttler H.R. and Gerdes V.E. Thyroid dysfunction and effects on coagulation and fibrinolysis: a systematic review. J. Clin. Endocrinol. Metab. 2007; 92: 2415-2420.

18. Hunt S.A., Abraham W.T., Chin M.H. et al. ACC/AHA 2005 guideline update for the diagnosis and management of chronic heart failure in the adult a report of the American College of Cardiology/American Heart Association Task Force on Practice Guidelines (Writing Committee to Update the 2001 Guidelines for the Evaluation and Management of Heart Failure): developed in collaboration with the American College of Chest Physicians and the International Society for Heart and Lung Transplantation: endorsed by the Heart Rhythm Society. Circulation. 2005; 112: 154-235.

19. Chen S., Shauer A., Zwas D.R. et al. The effect of thyroid function on clinical outcome in patients with heart failure. Eur J Heart Fail. 2014; 16: 217-226.

20. Ning Ning M.M., Dengfeng Gao, Vincenzo Triggiani, Massimo lacoviello. Et al. Prognostic Role of Hypothyroidism in Heart Failure. A Meta-Analysis. Cardiac resynchronization therapy. Am J Cardiol. 2015; 94(30): e1159.

21. Alessandro Pingitore and Giorgio lervasi. Triiodothyronine (T3) Effects on Cardiovascular System in Patients with Heart Failure. Institute of Clinical Physiology. 2008.Recent Patents on Cardiovascular Drug Discovery 3(1):19-27.

22. Paterson D. I., O'Meara E., Chow BJ., Ukkonen H., Beanlands RS. Recent advances in cardiac imaging for patients with heart failure. Curr Opin Cardiol. 2011; 26: 132143. 
23. Grais I.M., Sowers J.R. Thyroid and the heart. Am J Med. 2014; 127: 691-698.

24. Biondi B. Mechanisms in endocrinology: heart failure and thyroid dysfunction. Eur J Endocrinol. 2012; 167: 609-618.

25. Shah A.M., Mann D.L. In search of new therapeutic targets and strategies for heart failure: recent advances in basic science. Lancet. 2011; 378: 704-712.

26. Mitchell J.E, Hellkamp A.S, Mark D.B. et al. Thyroid function in heart failure and impact on mortality. JACC Heart Fail. 2013; 1: 48-55.

27. Perez A.C, Jhund P.S, Stott D.J. et al. Thyroid-stimulating hormone and clinical outcomes: the CORONA trial (controlled rosuvastatin multinational study in heart failure). JACC Heart Fail. 2014; 2: 35-40.

28. Razvi S., Shakoor A., Vanderpump M. et al. The influence of age on the relationship between subclinical hypothyroidism and ischemic heart disease: a meta-analysis. J Clin Endocrinol Metab. 2008; 93: 2998-3007.

29. Ochs N., Auer R., Bauer D.C. et al. Meta-analysis: subclinical thyroid dysfunction and the risk for coronary heart disease and mortality. Ann Intern Med. 2008;148:832-845.
30. Rodondi N., Elzen W.P., Bauer D.C. et al. Subclinical hypothyroidism and the risk of coronary heart disease and mortality. JAMA. 2010; 304: 1365-1374.

31. Kevin Ro, Alexander D. Yuen, Lin Du, Clarissa C. Ro, Christian Seger,Michael W. Yeh, Angela M. Leung, Connie M. Rhee. Impact of Hypothyroidism and Heart Failure on Hospitalization Risk. Thyroid. 2018; 28(9): 1094-1100.

32. Lakshmi Kannan, Pamela A. Shaw, Michael P. Morley, Jeffrey Brandimarto, James C. Fang, Nancy K. Sweitzer, Thomas P. Cappola, Anne R. Cappola. Thyroid Dysfunction in Heart Failure and Cardiovascular Outcomes. Circ Heart Fail. 2018;11: 1-9.

33. Provotorov V.M., Budnevskiy A.V., Semenkova G.G., Shishkina E.S. Proinflammatory cytokines in combination of coronary heart disease and chronic obstructive pulmonary disease. Klinicheskaia meditsina, 2015; 93(2): 5-9.

34. Budnevsky A.V., Ovsyannikov E.S., Labzhania N.B. Terapevticheskii Arkhiv, 2017; 89(1): 123-127.

35. Shiryaev O. Yu.; Yankovskaya V. L.; Budnevsky A. V. International journal of biomedicine. 2017; 7(3): 248-250 